\title{
Utility of diastolic dyssynchrony in the setting of cardiac resynchronization therapy
}

\author{
Marat Fudim, MD, and Salvador Borges-Neto, $\mathrm{MD}^{\mathrm{a}}$ \\ a Duke University Medical Center, Duke University, Durham, NC
}

Received Aug 30, 2019; accepted Aug 30, 2019

doi: $10.1007 / \mathrm{s} 12350-019-01889-4$

\section{See related article, https://doi.org/10.10 07/s12350-019-01845-2}

The definition of left ventricular mechanical dyssynchrony (LVMD) is the difference in the timing of mechanical contraction (systole) or relaxation (diastole) of the left ventricular wall segments. Notably, mechanical dyssynchrony does not equal electrical dyssynchrony, which is measured by QRS duration. Mechanical and electrical dyssynchrony do commonly occur simultaneously, but not always. ${ }^{1,2}$ To date, electrical dyssynchrony plays a greater role in the clinical management of heart failure patients given a clear association of a wide left bundle branch block $(>120 \mathrm{~ms})$ with adverse cardiovascular outcomes. More importantly, treatment of electrical dyssynchrony with cardiac resynchronization therapy (CRT) has been proven again and again to improve clinical outcomes. The role of LVMD has now been under investigation for over 2 decades. ${ }^{3}$ We now know that LVMD has significant prognostic implications across a variety of disease states. For example, in patients with heart failure and reduced ejection fraction (HFrEF) and no history of myocardial infarction, patients with LVMD had higher risks of cardiac events irrespective of QRS width and left ventricular ejection fraction (LVEF). ${ }^{4}$ In patients with coronary artery disease, LVMD was associated with mortality independent of electrical dyssynchrony. ${ }^{5,6}$ These findings were reproduced across other retrospective studies using ischemic and non-ischemic heart failure populations. ${ }^{7-9}$

\footnotetext{
Reprint requests: Marat Fudim, MD, Duke University Medical Center, Duke University, 2301 Erwin Road, Durham, NC 27710; marat.fudim@gmail.com

J Nucl Cardiol 2021;28:2644-6.

$1071-3581 / \$ 34.00$

Copyright (c) 2019 American Society of Nuclear Cardiology.
}

It has been suggested that lack of response to CRT meeting otherwise traditional criteria (LVEF $\leq 35 \%$ and electrical dyssynchrony) may be due to the absence of LVMD. ${ }^{10,11}$ Presence of LVMD in the setting of a non-left bundle branch block was associated with better long-term survival than absence of LVMD. ${ }^{11}$ Further, reversal of ventricular remodeling, a key feature of CRT, was directly linked to successful reduction on LVMD. ${ }^{12,13}$

Given the high CRT failure rate of around 30-40\% (lack of improvement in clinical symptoms and cardiac function ${ }^{14}$ ) and the potentially independent clinical significance of LVMD, the research into LVMD and CRT patient selection and post-CRT outcomes is gaining momentum. ${ }^{15}$ What if targeting mechanical dyssynchrony could improve the chance of successful CRT?

Nuclear imaging by Gated Single Photon Emission Computed Tomography (GSPECT) Myocardial Perfusion Imaging (MPI) now allows evaluating systolic and diastolic LVMD. Diastolic LVMD has been overlooked for a long period of time. Diastolic LVMD was found to have a stronger association with all-cause mortality than systolic LVMD in patients with coronary artery disease. Further, diastolic LVMD was associated with all-cause and cardiovascular mortality independent of LVEF. ${ }^{16}$ Finally, the few studies that evaluated the prognostic significance of diastolic LVMD via GSPECT MPI suggested a greater value of diastolic over systolic LVMD in selecting CRT candidates among HF patients. ${ }^{17}$

The analysis by Alexanderson-Rosas et al. assessed the utility of diastolic LVMD measured by GSPECT MPI in patients with HFrEF who underwent CRT. The secondary analysis of the VISION-CRT study sponsored by the International Atomic Energy Agency (IAEA) was a multinational prospective study. LVMDD (diastolic but also systolic) were measured before CRT device implantation. Patients were followed up for 6 months. Clinical improvement was defined as improvement in at least one of the following: decrease in $\geq 1$ NYHA 
functional class, improvement in LVEF (\%) by $\geq 5 \%$, reduction of left ventricular systolic volume by $\geq 15 \%$, and improvement in at least 5 points in Minnesota living with heart failure questionnaire. Diastolic LVMD was defined as diastolic phase standard deviation $\geq 40^{\circ}$.

Of 160 subjects with complete baseline information 20 died before the 6-month visit. Clinical improvement was seen in most patients. NYHA class improvement was seen in over $60 \%$ and LVEF improvement by $\geq 5 \%$ was seen in nearly $50 \%$. Nearly $83 \%$ of patients had improvement in at least one of the pre-defined clinical outcomes. Baseline diastolic LVMD was found in nearly $51 \%$ of patients at baseline, with a significant reduction to $32 \%$ at follow-up. The prevalence of systolic LVMD was reduced from $78 \%$ to $58 \%$.

Notably, at baseline, diastolic LVMD correlated moderately with structural and functional cardiac parameters such as LVEF, and left ventricular systolic and diastolic volume. Yet, in a regression analysis, no significant association between any diastolic dyssynchrony and change in clinical outcomes were found. This was the case for the combination or individual clinical endpoints. Similar findings were observed for systolic LVMD.

Some key take-aways from this study are as follows: 1. Prevalence of LVMD (diastolic or systolic) is high; 2. Diastolic and systolic LVMD can occur independently of each other; 3 . In concordance with prior evidence, diastolic and systolic LVMD are associated with progressive cardiac functional and structural remodeling. However, the major take-away and the most surprising finding of this analysis is the fact that presence of LVMD (irrespective of phase of cardiac cycle) was not associated with clinical outcomes. This finding casts a shadow on the concept that LVMD could be of either predictive value for CRT outcomes or be used as a selection tool for CRT candidates. The analysis of diastolic LVMD is of particular interest given that this represents the largest analysis of diastolic LVMD using GSPECT MPI in the setting of CRT. A hint towards a lack of association of diastolic LVMD with outcomes was recently demonstrated in separate cohort of patients with predominant heart failure with preserved ejection fraction. ${ }^{18}$ Does this mean, there is no value in measuring diastolic (also systolic) LVMD and consider them potential therapeutic targets? We don't think so. The study results need to be viewed with some caution given some notable limitations. First, this was a small retrospective analysis. Second, the analysis strictly evaluated the association of baseline LVMD with a change in clinical parameters (outcomes) and not a change in LVMD. This type of an analysis would provide an important insight into the true dynamic of LVMD and clinical changes with CRT. Also, it is important to understand whether CRT affected LVMD in patients with clinical improvement. This question is currently not answered by this analysis. Finally, it is important to acknowledge that follow-up ended at 6 months. While changes beyond 6 months are unlikely, future studies should explore the changes in LVMD and clinical outcomes beyond 6 months.

In summary, whether LVMD can be used to improve CRT candidate selection is unclear. The available mixed data demand a prospective clinical trial of CRT eligible patients utilizing GSPECT MPI data and clinical data to study outcomes.

\section{Disclosure}

Salvador Borges-Neto is supported by a GE Healthcare grant. Marat Fudim has no conflict of interest.

\section{References}

1. Fudim M, Borges-Neto S. A troubled marriage: When electrical and mechanical dyssynchrony don't go along. J Nucl Cardiol 2018;26:1240-2.

2. Yu CM, Lin H, Zhang Q, Sanderson JE. High prevalence of left ventricular systolic and diastolic asynchrony in patients with congestive heart failure and normal QRS duration. Heart 2003;89:54-60.

3. Pagnanelli R, Fudim M, Borges-Neto S. Technologist corner: Value of radionuclide ventriculography to assess mechanical dyssynchrony and predict the cardiac resynchronization therapy response. J Nucl Cardiol 2016;23:491-2.

4. Bader H, Garrigue S, Lafitte S, Reuter S, Jais P, Haissaguerre M, Bonnet J, Clementy J, Roudaut R. Intra-left ventricular electromechanical asynchrony: A new independent predictor of severe cardiac events in heart failure patients. J Am Coll Cardiol 2004;43:248-56.

5. Hess PL, Shaw LK, Fudim M, Iskandrian AE, Borges-Neto S. The prognostic value of mechanical left ventricular dyssynchrony defined by phase analysis from gated single-photon emission computed tomography myocardial perfusion imaging among patients with coronary heart disease. J Nucl Cardiol 2017;24:48290.

6. Fudim M, Fathallah M, Shaw LK, Liu PR, James O, Samad Z, Piccini JP, Hess PL, Borges-Neto S. The prognostic value of diastolic and systolic mechanical left ventricular dyssynchrony among patients with coronary heart disease. JACC Cardiovasc Imaging 2019;12:1215-26.

7. AlJaroudi W, Alraies MC, Menon V, Brunken RC, Cerqueira MD, Jaber WA. Predictors and incremental prognostic value of left ventricular mechanical dyssynchrony response during stress-gated positron emission tomography in patients with ischemic cardiomyopathy. J Nucl Cardiol 2012;19:958-69.

8. Goldberg AS, Alraies MC, Cerqueira MD, Jaber WA, Aljaroudi WA. Prognostic value of left ventricular mechanical dyssynchrony by phase analysis in patients with non-ischemic cardiomyopathy with ejection fraction $35-50 \%$ and QRS $<150 \mathrm{~ms}$. J Nucl Cardiol 2014;21:57-66.

9. Pazhenkottil AP, Buechel RR, Husmann L, Nkoulou RN, Wolfrum M, Ghadri JR, Kummer J, Herzog BA, Kaufmann PA. Long- 
term prognostic value of left ventricular dyssynchrony assessment by phase analysis from myocardial perfusion imaging. Heart 2011;97:33-7.

10. Emkanjoo Z, Esmaeilzadeh M, Mohammad Hadi N, Alizadeh A, Tayyebi M, Sadr-Ameli MA. Frequency of inter- and intraventricular dyssynchrony in patients with heart failure according to QRS width. Europace 2007;9:1171-6.

11. Perry R, De Pasquale CG, Chew DP, Aylward PE, Joseph MX. QRS duration alone misses cardiac dyssynchrony in a substantial proportion of patients with chronic heart failure. J Am Soc Echocardiogr 2006;19:1257-63.

12. Yu CM, Chau E, Sanderson JE, Fan K, Tang MO, Fung WH, Lin H, Kong SL, Lam YM, Hill MR, Lau CP. Tissue Doppler echocardiographic evidence of reverse remodeling and improved synchronicity by simultaneously delaying regional contraction after biventricular pacing therapy in heart failure. Circulation 2002; 105:438-45.

13. Stellbrink C, Breithardt OA, Franke A, Sack S, Bakker P, Auricchio A, Pochet T, Salo R, Kramer A, Spinelli J, Investigators P-C, Group CPIGCHFR. Impact of cardiac resynchronization therapy using hemodynamically optimized pacing on left ventricular remodeling in patients with congestive heart failure and ventricular conduction disturbances. J Am Coll Cardiol 2001;38:1957-65.

14. Bertini M, Hoke U, van Bommel RJ, Ng AC, Shanks M, Nucifora G, Auger D, Borleffs CJ, van Rijnsoever EP, van Erven L, Schalij MJ, Marsan NA, Bax JJ, Delgado V. Impact of clinical and echocardiographic response to cardiac resynchronization therapy on long-term survival. Eur Heart $\mathbf{J}$ Cardiovasc Imaging 2013;14:774-81.

15. Fudim M, Dalgaard F, Fathallah M, Iskandrian AE, Borges-Neto S. Mechanical dyssynchrony: How do we measure it, what it means, and what we can do about it. J Nucl Cardiol 2019. https://d oi.org/10.1007/s12350-019-01758-0.

16. Fudim M, Fathallah M, Shaw LK, Liu PR, James O, Samad Z, Piccini JP, Hess PL, Borges-Neto S. The prognostic value of diastolic and systolic mechanical left ventricular dyssynchrony among patients with coronary heart disease. JACC Cardiovasc Imaging 2018. https://doi.org/10.1016/j.jcmg.2018.05.018.

17. Ando M, Yamamoto T, Saiki Y, et al. Is diastolic dyssynchrony more important determinant than systolic dyssynchrony for the cardiac resynchronization therapy (CRT)? A study using gated myocardial perfusion SPECT. Circulation 2016;120:S859.

18. Fudim M, Fathallah M, Shaw LK, James O, Samad Z, Piccini JP, Hess PL, Borges-Neto $\mathrm{S}$. The prognostic value of diastolic and systolic mechanical left ventricular dyssynchrony among patients with coronary artery disease and heart failure. J Nucl Cardiol 2019. https://doi.org/10.1007/s12350-019-01843-4.

Publisher's Note Springer Nature remains neutral with regard to jurisdictional claims in published maps and institutional affiliations. 